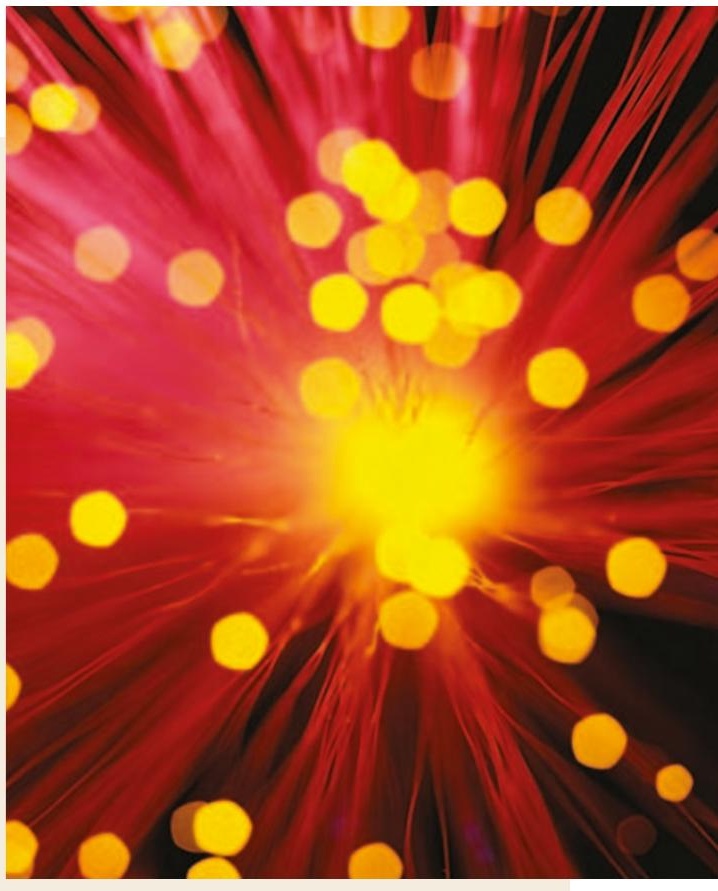

Saltatory conduction - the process by which action potentials propagate along myelinated nerves - depends on the fact that voltagegated $\mathrm{Na}^{+}$channels form clusters at the nodes of Ranvier, between sections of the myelin sheath. New findings from Eshed et al. show that Schwann cells produce a protein called gliomedin, and that this is responsible for the clustering of these channels.

The formation of the nodes of Ranvier is specified by the myelinating cells, not the axons, and an important component of this process in the peripheral nervous system is the extension of microvilli by Schwann cells. These microvilli contact the axons at the nodes, and it is here that the Schwann cells express the newly discovered protein gliomedin.

Eshed et al. showed that gliomedin is a ligand for two axonal cell adhesion molecules - neurofascin and NrCAM - that are found at the nodes of Ranvier, where they interact with $\mathrm{Na}^{+}$channels. When the authors either disrupted the localization of gliomedin by using a soluble fusion protein that contained the extracellular domain of neurofascin, or used RNA interference to suppress the expression of gliomedin, the characteristic clustering of $\mathrm{Na}^{+}$channels at the nodes of Ranvier did not occur.

Aggregation of the domain of gliomedin that binds neurofascin and NrCAM on the surface of purified neurons also caused the clustering of neurofascin, $\mathrm{Na}^{+}$channels and other nodal proteins. These findings support a model in which gliomedin on Schwann cell microvilli binds to neurofascin and $\mathrm{NrCAM}$ on axons, causing them to cluster at the nodes of Ranvier, and leading to the formation of complexes that contain $\mathrm{Na}^{+}$channels.

Rachel Jones

\section{(2) References and links}

ORIGINAL RESEARCH PAPER Eshed, Y. et al. Gliomedin mediates Schwann cell-axon interaction and the molecular assembly of the nodes of Ranvier. Neuron 47, 215-229 (2005)

FURTHER READING Sherman, D. L. \& Brophy, P. J.

Mechanisms of axon ensheathment and myelin growth. Nature Rev. Neurosci. 6, 683-690 (2005)

\title{
Long-distance communication
}

Traditionally, we have viewed neurotransmitter release as being strictly confined to presynaptic active zones and postsynaptic densities. New work by Coggan, Bartol and colleagues, using a combination of high-resolution electron microscopy and computational simulations, indicates that additional neurotransmitter release distant from these sites (ectopic neurotransmitter release) is necessary to activate certain receptors.

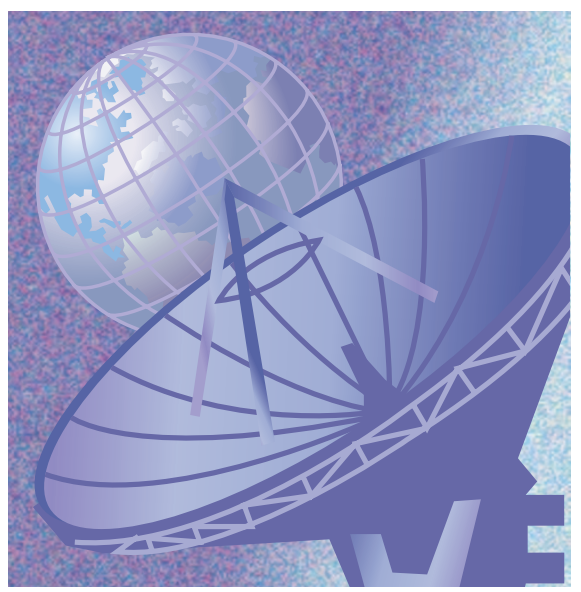

Recent work in the chick ciliary ganglion has shown that one class of nicotinic acetylcholine receptor (nAChR) - the $\alpha 7-n A C h R s$, which are located on spines - is virtually absent from the postsynaptic density. Nevertheless, these receptors account for numerous changes in physiological measurements, which indicates that they must be involved in neurotransmitter responses. Furthermore, electron microscopy has shown presynaptic vesicles that are ready for neurotransmitter release and fusing vesicles at locations distant from the postsynaptic density. However, until now, there has been no experimental confirmation of ectopic neurotransmitter release during synaptic transmission.

Coggan, Bartol and co-workers used electron microscopy studies to create a geometrically accurate three-dimensional model of a ciliary ganglion synapse, and, on the basis of previous work, incorporated into the model information about the distribution and kinetic properties of presynaptic release sites, acetylcholinesterase and $\alpha 7$-nAChRs. They simulated miniature excitatory postsynaptic currents (mEPSCs) by imitating vesicle release at numerous sites, including postsynaptic density and non-postsynaptic density regions, while simultaneously varying the amount of acetylcholine released into the synaptic cleft.

The simulated mEPSCs most closely resembled previously recorded mEPSCs from a ciliary ganglion in situ when the model included a high proportion of neurotransmitter release that occurred away from the postsynaptic density. By contrast, simulations in which vesicle fusion was limited to the postsynaptic density, or when $\alpha 7$-nAChRs were included in the postsynaptic density, elicited mEPSCs that were at odds with the experimental recordings.

This intriguing finding challenges previous assumptions about the structure and function of synapses. So far, the reason for ectopic neurotransmitter release specifically in the ciliary ganglion is unclear, but the authors speculate that it might relate to the specialized molecular and physiological properties of $\alpha 7$-nAChRs. It will be interesting to determine whether this mechanism for neurotransmitter release is exclusive to the ciliary ganglion or whether it occurs during the activation of other types of receptor.

Alison Rowan

6) References and links ORIGINAL RESEARCH PAPER Coggan, J. S. \& Bartol, T. M. et al. Evidence for ectopic neurotransmission at a neuronal synapse. Science $\mathbf{3 0 9}, 446-451$ (2005) 